\title{
Is Oxidative Stress an Effective Risk Factor in the Development of Overt Hypothyroidism in Hashimoto Thyroiditis?
}

$\underline{\text { İhsan Ates }}{ }^{1}$, Mustafa Altay ${ }^{1}$, Fatma Meriç Yılmaz ${ }^{2}$, Nisbet Y1lmaz ${ }^{1}$, Serdar Güler ${ }^{3}$

${ }^{1}$ Ankara Numune Education and Research Hospital, Department of Internal Medicine, Ankara, Turkey

${ }^{2}$ Ankara Numune Education and Research Hospital, Department of Biochemistry, Ankara, Turkey

${ }^{3}$ Ankara Numune Education and Research Hospital, Department of Endocrinology and Metabolism, Ankara, Turkey

\section{Objectives:}

\section{Methods:}

\section{Results:}

Conclusions:

Higher levels of autoantibodies and oxidative stress in patients developing overt hypothyroidism shows that oxidative stress plays a major role in the pathogenesis and progression of autoimmune thyroiditis.

Eighty patients (40 euthyroid and 40 subclinical hypothyroidism) older than 18 years with newly diagnosed Hashimoto thyroiditis and not yet on any treatment were enrolled. Patients were followed up for 9 months. Blood sample was drawn at diagnosis for thyroid function tests and oxidative stress parameters. Total antioxidant status (TAS), total oxidative status (TOS), paraoxonase 1 (PON1), arylesterase and total thiol (total $\mathrm{SH}$ ) were measured by colorimetric method. The ratio of serum TOS level and TAS level gave the oxidative stress index. Thyroid stimulating hormone (TSH), free thyroxine (fT4), anti-thyroid peroxidase (anti-TPO) and anti-thyroglobulin (anti-Tg) were measured by ECLIA method

Total SH level was found to be higher in the euthyroid group as compared to the subclinical hypothyroidism group $(p=0.019)$. TAS, TOS, OSI, PON and arylesterase levels were similar in the two groups $(p>0.05)$. At nine months of follow-up, overt hypothyroidism developed in 14 patients. Patients with overt hypothyroidism tended to have significantly higher average TOS and OSI levels at diagnosis $(p<0.001)$ and significantly higher anti-Tg and anti-TPO levels at hypothyroidism development $(p<0.05)$ than those who did not develop overt hypothyroidism. Multivariable Cox regression analysis showed TSH level $(H R=1.348, p<0.001)$, fT4 level $(H R=0.481, p=0.017)$ and OSI ratio $(H R=2.349, p<0.001)$ to be independent predictors for the development of overt hypothyroidism in euthyroid patients and those with subclinical hypothyroidism.

\section{References:}

1. Phillips, D., et al., Autosomal dominant transmission of autoantibodies to thyroglobulin and thyroid peroxidase. J Clin Endocrinol Metab, 1990. 70(3): p. 742-6.

2. Rostami, R., et al., Enhanced oxidative stress in Hashimoto's thyroiditis: inter-relationships to biomarkers of thyroid function. Clin Biochem, 2013. 46(4-5): p. 308-12.

3. Bagchi, N., et al., Antioxidants delay the onset of thyroiditis in obese strain chickens. Endocrinology, 1990. 127(4): p. 1590-5. 